Discrete Comput Geom 26:573-590 (2001)

DOI: $10.1007 / \mathrm{s} 00454-001-0047-6$

\title{
Hard Tiling Problems with Simple Tiles*
}

\author{
C. Moore ${ }^{1,2,3}$ and J. M. Robson ${ }^{4}$ \\ ${ }^{1}$ Computer Science Department, University of New Mexico, \\ Albuquerque, NM 87131, USA \\ ${ }^{2}$ Department of Physics and Astronomy, University of New Mexico, \\ Albuquerque, NM 87131, USA \\ ${ }^{3}$ Santa Fe Institute, \\ 1399 Hyde Park Road, Santa Fe, NM 87501, USA \\ moore@santafe.edu \\ ${ }^{4}$ LaBRI, Université Bordeaux 1, \\ 351 Cours de la Libération, 33405 Talence, France \\ robson@labri.u-bordeaux.fr
}

\begin{abstract}
It is well known that the question of whether a given finite region can be tiled with a given set of tiles is NP-complete. We show that the same is true for the right tromino and square tetromino on the square lattice, or for the right tromino alone. In the process we show that Monotone 1-in-3 Satisfiability is NP-complete for planar cubic graphs. In higher dimensions we show NP-completeness for the domino and straight tromino for general regions on the cubic lattice, and for simply connected regions on the four-dimensional hypercubic lattice.
\end{abstract}

\section{Introduction}

Tilings of the plane have long intrigued statistical physicists, computer scientists and recreational mathematicians. If we have as many copies as we like of a finite set of shapes, can we fill a given region with them? Tiling problems are potentially very hard: in fact, telling whether a finite set of tiles can tile the infinite plane is undecidable, since the non-existence of a tiling is equivalent to the Halting Problem [1], [2]. For finite regions, this problem becomes NP-complete [3], meaning that it is just as hard as combinatorial search problems like Traveling Salesman or Hamiltonian Path [4].

* This work was partially supported by the Sandia University Research Program. 
In fact, tiling problems can be difficult even for small sets of very simple tiles, such as polyominoes [5] with as few as three cells. Specifically, Beauquier et al. [6] showed that for general regions of the square lattice the tiling problem is NP-complete for horizontal dominoes and vertical trominoes, and for any other pair of bars where either has length greater than 2 . This includes the straight tromino alone when rotations are allowed. For simply connected regions in the plane, on the other hand, Kenyon and Kenyon [7] provided a linear-time algorithm for the problem with any pair of bars. When rotations are allowed, Rémila provided a polynomial-time algorithm for bars of length 2 and 3 in two dimensions, both in simply connected regions [8] and in general [9].

In this paper we give some additional examples of this kind. For the right tromino and square tetromino, we show that tiling a region on the square lattice is NP-complete, and that the problem of counting how many tilings exist is $\mathbf{\# P - c o m p l e t e , ~ m a k i n g ~ i t ~ a s ~ h a r d ~}$ as enumeration problems like calculating the permanent of a matrix [10]. For the right tromino alone, we show NP-completeness using a more complicated construction based on 1-in-3 Satisfiability for planar cubic graphs, but unfortunately we have no result on the counting problem.

In higher dimensions we consider the domino and straight tromino with rotations allowed. We show that this tiling problem is NP-complete for general regions in the cubic lattice, and for simply connected regions in the four-dimensional hypercubic lattice.

We end with a discussion of some open problems, and a discussion of whether these results could be tightened further.

\section{Building Circuits with Tiles}

We call the question of whether a given set of shapes can tile a given finite subset of the square lattice, and how many such tilings exist, the existence and counting problems. These problems are trivially in NP and \#P respectively, since we can confirm that a proposed tiling works in polynomial time. In this section we consider the set of tiles shown in Fig. 1, namely the right tromino and square tetromino, with rotations allowed.

While the first hardness results about tiling relied on simulating steps of a Turing machine from row to row, here we instead simulate Boolean circuits, where "wires" with two possible tilings carry truth values, and junctions in these wires simulate logical gates. Similar approaches are taken in [6], [9], and [11]-[13]. The question of whether a tiling exists then corresponds to the canonical NP-complete problem Satisfiability, which asks whether a set of truth values for the inputs exists that makes the output true [4].

Our wire is shown in Fig. 2. It moves in knight's moves in eight possible directions across the lattice. Given an orientation of the wire leading from input to output, the tilings

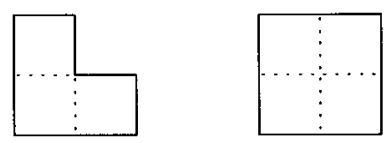

Fig. 1. The right tromino and square tetromino. 


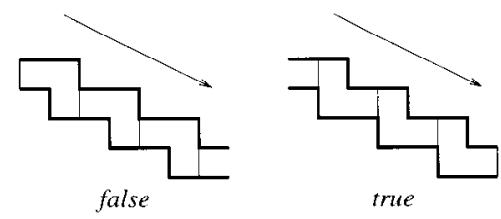

Fig. 2. The wire for tilings by the right tromino and square tetromino. Each wire contains a sequence of $3 \times 1$ rectangles offset by a knight's move and is given an orientation; we define its value as true or false depending on whether the second cell of a rectangle is in the same tromino as the first or the last.

where cells in between jogs are occupied by trominoes upstream and downstream from them will be considered "true" and "false," respectively.

Given this encoding of truth values in wires, Fig. 3 shows an AND gate. Each pair of truth values for the inputs $a$ and $b$ has one and only one tiling, which causes the output to have the value $a \wedge b$. Figure 4 shows a NOT gate, where the output has the opposite truth value from the input. By combining AND and NOT gates we can generate any Boolean function, including a "crossover gadget" that allows us to cross wires in the plane [14]. Since we may want to use the output of one gate as the input of several others, Fig. 5 shows how to split a wire into two copies with the same truth value. To create our inputs and outputs, we need to be able to start variable wires with either truth value, and end wires in a way that requires them to be true. Figure 6 shows how to do both of these.

There are two more subtleties that must be dealt with. First, it must be possible to bend wires among their eight possible orientations. We can do this by rotating and reflecting a wire's direction as in Fig. 7; these turns are derived simply by setting one of an AND gate's inputs to be true. Secondly, a wire of a given orientation has a knight's move periodicity, so that if we color the cells of the lattice as in Fig. 8 each step can only land on one of the five colors. This gives each wire a kind of phase, and the zigzag shown in the figure allows us to change this phase so that a wire's truth value can be delivered to cells of any color. Then the output of any gate can become the input of any other, as long
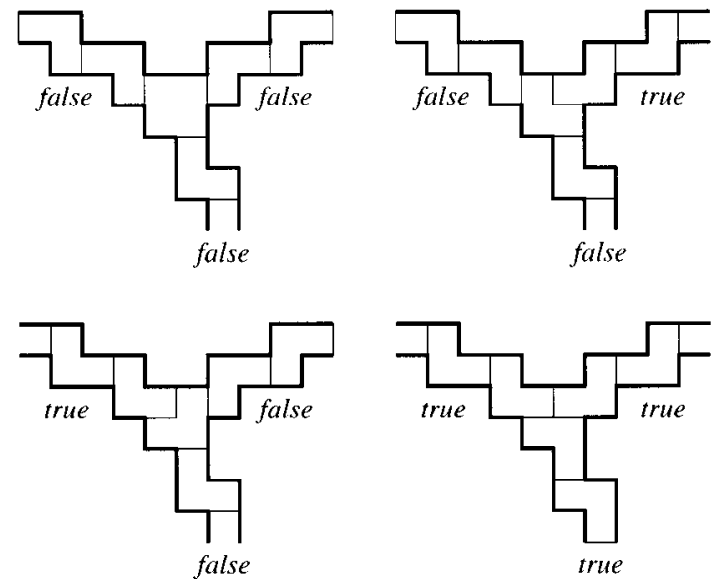

Fig. 3. Our AND gate. Two input wires enter a $2 \times 2$ square from above, and the output exits from below. For each set of truth values for the inputs, there is exactly one tiling as shown. 

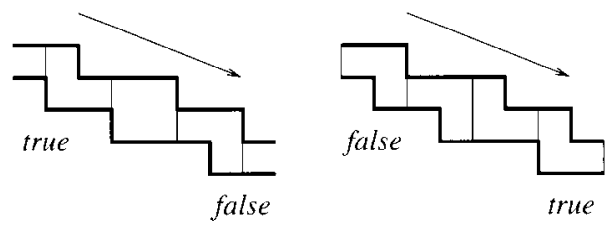

Fig. 4. Our NOT gate, which negates the signal along a wire.
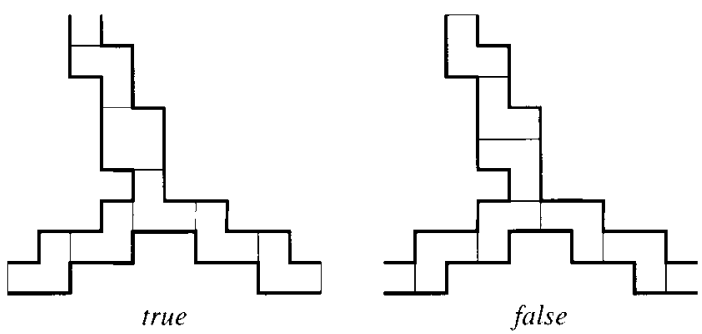

Fig. 5. How to split a wire (entering from above) into two copies with the same truth value.

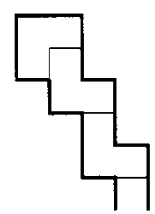

false

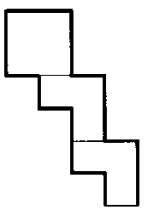

true

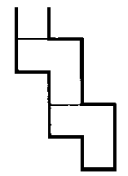

true

Fig. 6. Our variables. The bulb at the top can be tiled in two ways, which produce a true or false value on the wire it's connected to. On the right, how to end a wire so that it must be true for a tiling to exist.
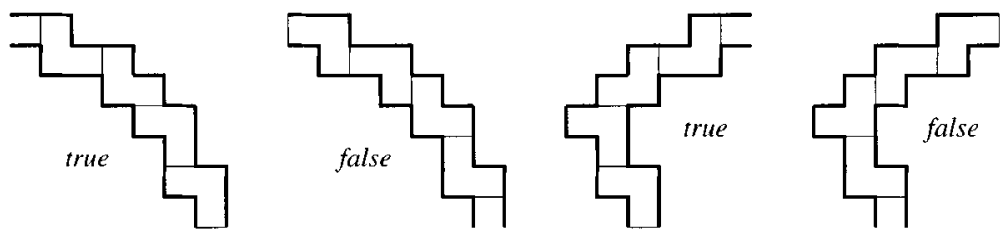

Fig. 7. How to bend wires. On the left the wire's direction is reflected around a diagonal line, and on the right it is rotated $90^{\circ}$. These allow us to change from any of the eight directions to any other.
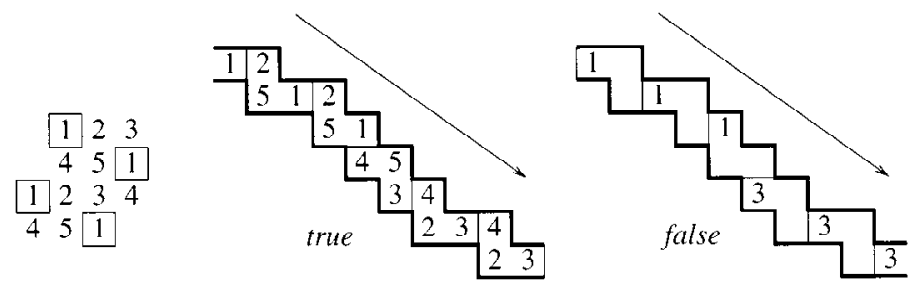

Fig. 8. Changing the phase of a wire. The zigzag shown keeps the truth value the same, but cycles among the five colors of the lattice. 
as the gates are separated widely enough. It is easy to show that this separation need only be polynomially large.

It is clear from this construction that for any Boolean circuit, we can construct a region of the plane such that the right tromino and square tetromino can tile it if and only if the circuit is satisfiable. Moreover, this reduction is parsimonious, i.e. the mapping between solutions of the two problems is one-to-one, so the number of solutions is the same in both cases. Thus the question of whether any tilings exist, and how many there are, are equivalent to Satisfiability and \#Satisfiability, which are $\mathbf{N P}$-complete and \#P-complete respectively [4], [10].

We conclude this section with Fig. 9, an example of a figure produced by our reduction from the expression $\neg(\neg A \wedge \neg B) \wedge \neg(A \wedge B)$, or equivalently $A \neq B$. $A$ is the variable at the top and $B$ the one in the middle. To save space we have reduced the length of the

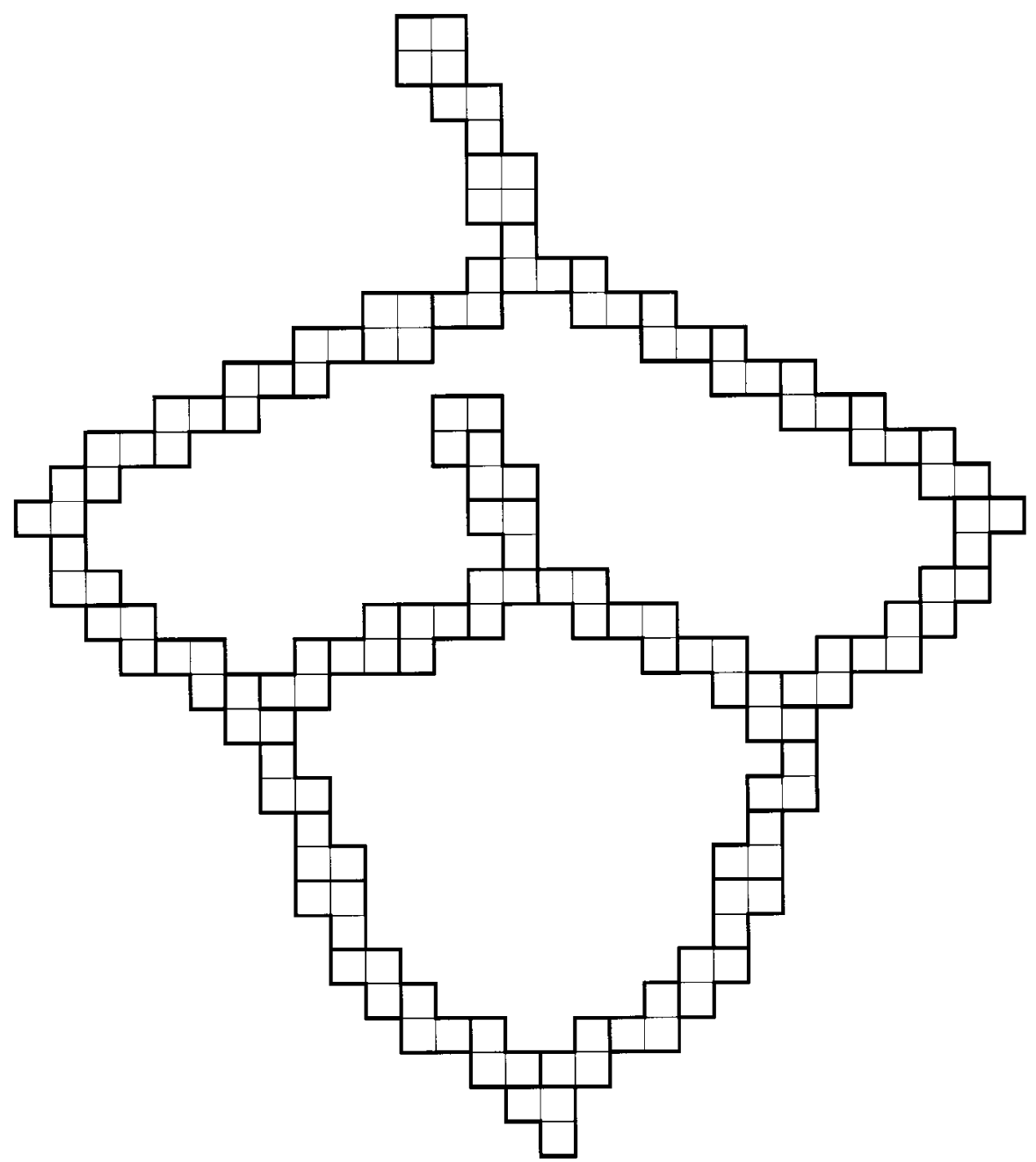

Fig. 9. A witness to the satisfiability of $\neg(\neg A \wedge \neg B) \wedge \neg(A \wedge B)$. 
wire from $B$ to its splitter to zero and we have made the components fit together without using explicit phase changes by adjusting the lengths of the sections of wire. The tiling shown is that for $A=$ true, $B=$ false.

\section{Right Trominoes Alone}

We now improve on the result of the previous section, and show that the existence problem is NP-complete for the set of tiles consisting only of the right tromino (with rotations allowed). The main reason that this is more difficult than for the tromino and square tetromino is that every tile covers three squares. If we use the same wires as before, where the truth value depends on the protrusion, or lack of it, of a single site, then we can only represent gates where the output is a linear function of the inputs mod 3. While this prevents us from coding Boolean circuits directly, we can still represent a version of Satisfiability which we will show is NP-complete.

First, we represent variables with nodes as in Fig. 10, in which three outgoing wires are constrained to be all true or all false.

Secondly, we represent clauses with the nodes of Fig. 11, which require that exactly one of three incoming wires be true, and that the other two be false. If exactly one wire is true, the tilings of the wires cover exactly one square of the central $2 \times 2$ square, leaving a space for one right tromino. Otherwise the number of squares left in the central square is not a multiple of 3 and so cannot be tiled by trominoes.

If we consider a bipartite graph of these two types of nodes connected with wires as in the previous section, then the tiling problem becomes a variant of 1-in-3 Satisfiability. That is, we have a set of variables and a set of three-variable clauses, and we want to know if there is an assignment of truth values to the variables such that exactly one of the three variables in each clause is true.

However, here we have a restricted case of this problem in which the expression's graph is planar, there is no negation, and each variable occurs in exactly three clauses since our variable nodes have three wires emanating from them. We claim that any bipartite planar cubic graph (with the two types of vertices being variables and 1-in3 clauses) can be transformed into a plane figure which is tileable if and only if the expression represented by the graph is satisfiable. We use new wire and corner subfigures as shown in Figs. 12 and 13. Note that we could have used these wires and corners in the
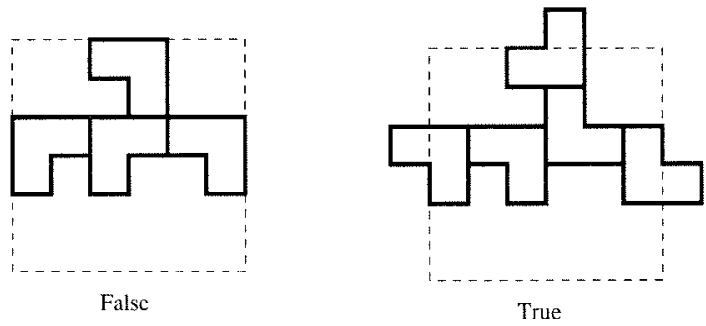

Fig. 10. A variable subfigure and its two possible tilings. 

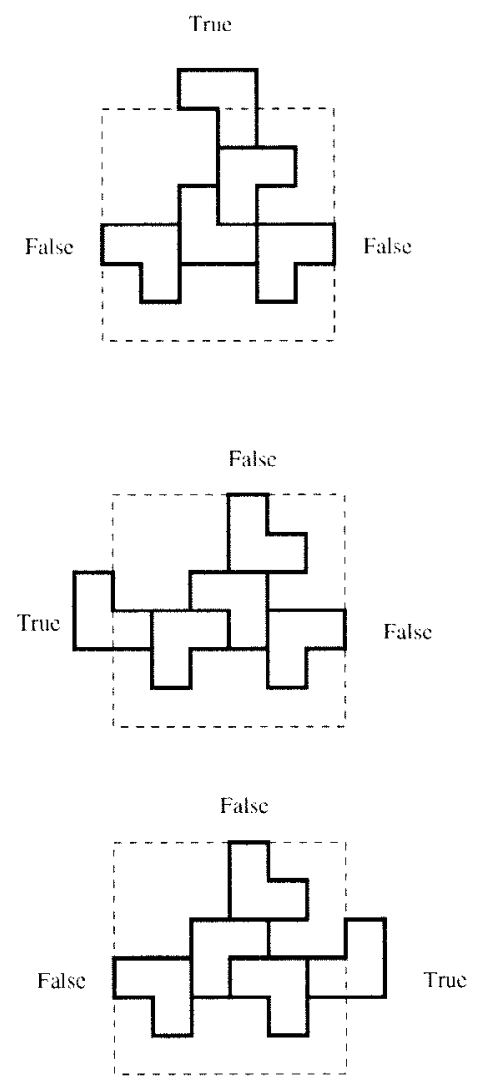

Fig. 11. A clause subfigure and its three possible tilings.
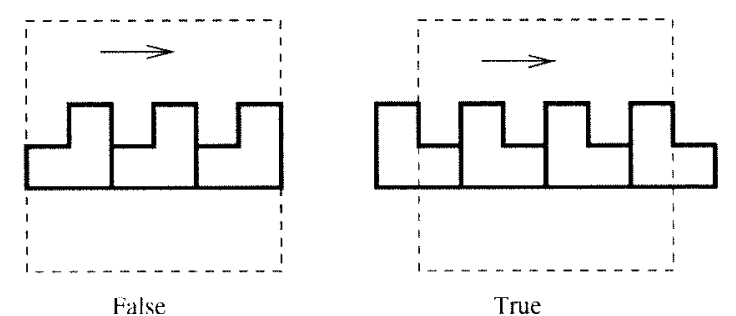

Fig. 12. A section of wire and its two possible tilings.

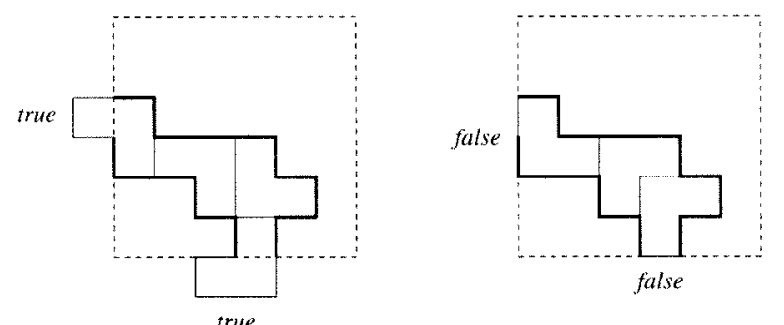

Fig. 13. A left-hand corner and its two possible tilings. 
previous reduction but the clause subfigure would have been incorrect in the case where tetrominoes were allowed. We proceed as follows:

- Choose a planar embedding.

- Find a drawing of the graph on a square grid so that vertices are at grid points and edges are disjoint paths along grid lines. Theorem 8.3 of [15] tells us that this can be done in linear time. Now every grid point either is a variable or clause vertex with edges leaving in three orthogonal directions, or has a wire passing through with or without a $90^{\circ}$ turn, or is empty.

- Replace every non-empty grid point by a $6 \times 6$ square of cells containing either a variable, a clause, a corner or a wire as shown in Figs. 10-13 rotated as necessary. The subfigures of Figs. 10-13 are all designed to fit into a $6 \times 6$ square (indicated by the dashed lines) so that, whenever a wire crosses from one square to another it occupies the two middle cells of the boundary of the upstream square and one of the two middle squares of the adjacent boundary of the downstream square but otherwise no cells on the periphery of squares are occupied. This suffices to guarantee that the signals can be transmitted along the wires as required and that no other tilings are possible. Note that we have shown all the gadgets so that wires exit $6 \times 6$ squares to their right of the center of the square's edge. In all cases we could reflect the tromino straddling the boundary to change to a "left exit" and still have a valid gadget; thus a left entry such as exists in the clause figure or in all the reflected versions causes no problems.

Thus, to complete our proof we need only show that Cubic Planar Monotone 1-in-3 Satisfiability is NP-complete, and we do this in the next section.

\subsection{Cubic Planar Monotone 1-in-3 Satisfiability}

Laroche [16] showed that Planar Monotone 1-in-3 SAT is NP-complete. This is the restriction of Monotone 1-in-3 SAT to expressions whose graph $G$ is planar, where $G$ is defined with a vertex for each variable and each clause of the expression, and an edge joining each clause to each variable appearing in it.

We will show that this problem remains NP-complete when restricted to expressions where every variable has exactly three occurrences, i.e. those whose graph is cubic. (An alternate formulation is to consider a hypergraph where each clause is a node and each variable is a hyperedge joining three nodes. Since each true variable "covers" three clauses and every clause must be covered exactly once, we can then regard this problem as Exact Cover by 3-Sets in the case where the associated hypergraph is planar.)

While the reduction from this version of SAT to the tiling problem we gave in the previous section is parsimonious, this does not show that the counting problem is \#Pcomplete, since we do not have such a result for Cubic Planar Monotone 1-in-3 SAT. We leave this as an open problem for the reader.

Our reduction is from unrestricted Planar Monotone 1-in-3 SAT. In the diagrams of this section we use the same convention as in [16] that circles represent variables and squares represent clauses. 
The reduction proceeds in two stages. The first stage produces an expression $E^{\prime}$ which is planar and satisfiable if and only if the original was, and where each variable has three or fewer occurrences. The second stage, rather more intricate, adds new clauses and auxiliary variables producing an expression $E^{\prime \prime}$ where all variables have exactly three occurrences.

\subsection{The Reduction}

We start by introducing some simple components to be used in the first stage. An equality verifier for two variables $v_{1}$ and $v_{2}$ consists of two new variables $x$ and $y$ together with the two clauses $\left(v_{1}, x, y\right)$ and $\left(v_{2}, x, y\right)$. Clearly this can be satisfied if and only if $v_{1}=v_{2}$; moreover, if $v_{1}$ and $v_{2}$ are false, then exactly one of $x$ and $y$ is true. A chain between two variables $v_{1}$ and $v_{2}$ consists of new variables $u$ and $w$ and three equality verifiers: one between $v_{1}$ and $u$, one between $u$ and $w$, and one between $w$ and $v_{2}$. Again a chain forces $v_{1}=u=w=v_{2}$ in any satisfying assignment and when $v_{1}$ and $v_{2}$ are false, the choice of $x$ or $y$ being true is independent for the three equality verifiers. A chain is shown in Fig. 14 .

Given an expression $E$ which is an instance of Planar Monotone 1-in-3 SAT, we choose a planar embedding of its graph $G$. For each variable $v_{i}$ that occurs in $k>1$ clauses, we replace it with a set of variables $v_{i j}$ for $j=1 \cdots k$, one for each occurrence. We then link these $k$ variables together with chains as in Fig. 15. We call the resulting expression $E^{\prime}$ and its planar graph $G^{\prime}$. Note that this sequence of links between the $v_{i j}$ is not completed to form a cycle; we omit an arbitrarily chosen link, say between $v_{i, 1}$ and $v_{i, k}$. Therefore, any boundary between two faces that includes a chain in fact includes chains from two different variables that appear in the same clause, a fact which we use below.

This completes the first stage of the reduction. Clearly $E^{\prime}$ is satisfiable if and only if $E$ was, and every variable has three or fewer occurrences. The variables with less than three occurrences are the $x$ and $y$ variables of each equality verifier, the intermediate $u$ and $w$ variables of each chain, the two unlinked variables $v_{i, 1}$ and $v_{i, k}$ for each $v_{i}$ with $k>1$ occurrences, and the original variables $v_{i}$ which have only one occurrence in $E$. The total number of missing occurrences is a multiple of 3 since each variable must have three occurrences and each clause absorbs three.

We now consider the faces of $G^{\prime}$. Our goal is to associate each missing occurrence to a face so that each face has a multiple of three additional occurrences along its boundary. There are some faces bordered by the $v_{1}, x, v_{2}, y$ of an equality verifier; we call these faces trivial, and assign no missing occurrences to them. The remaining assignments are made as follows.

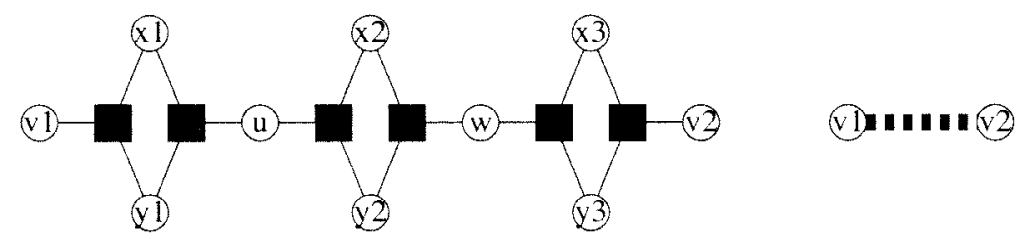

Fig. 14. A chain that forces two variables $v_{1}$ and $v_{2}$ to be equal, and our abbreviation for it. 


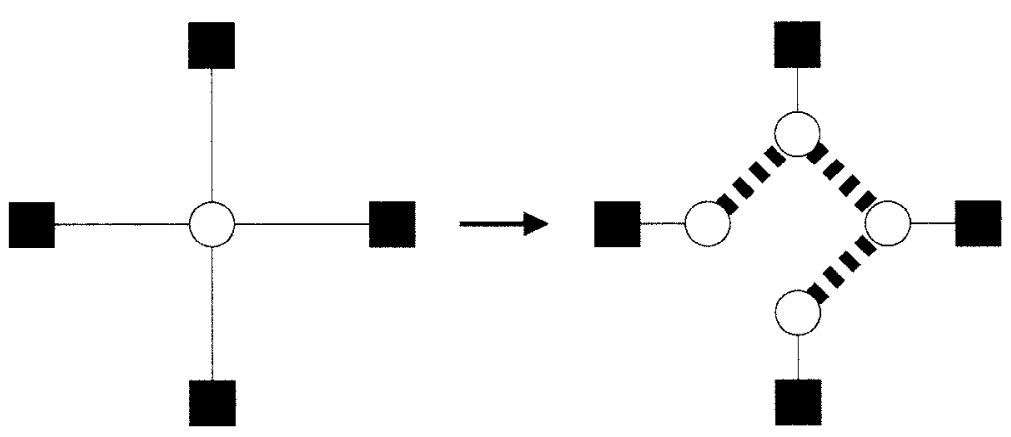

Fig. 15. Replacing variables with $k>1$ occurrences with $k$ variables connected by chains. One link is omitted arbitrarily, say between $v_{i, 1}$ and $v_{i, k}$, so as not to form a complete cycle.

Since the $x$ and $y$ variables of the chains have only one face adjacent to them, we start with each non-trivial face having a certain number of additional occurrences mod 3. We now note that every non-trivial face has at least one chain along its boundary. Each chain has two variables $u$ and $v$ with one occurrence each that can be assigned either to this face or to one adjacent to it. In this way, we can transfer 0,1 or $2(\bmod 3)$ additional occurrences from this face to the next one. If we define a tree of faces (i.e. a spanning tree of the dual graph) we can start at the leaves and change the number of additional occurrences in each face to $0 \bmod 3$, until we reach the root, where the number of occurrences left is also $0 \bmod 3$ since the total is a multiple of 3 .

We use a similar strategy to control, for each face, how many of the additional occurrences entering it are true, in extending a satisfying assignment for $E^{\prime}$ to one for $E^{\prime \prime}$. Recall that along the boundary between any pair of adjacent non-trivial faces are chains corresponding to two different variables that appear in the same clause. In any satisfying assignment, at least one of these is false, and so in each link of its chain we can take either $x$ or $y$ to be true. In this way, we can transfer 0,1 or $2(\bmod 3)$ true variables from one face to another. If we again define a tree of faces, we can start at the leaves and change the number of true additional occurrences in each face to $0 \bmod 3$, until we reach the root. At the root face, the number of true additional occurrences is simply the total mod 3. This is - (the number of clauses of $E^{\prime}$ ) $\bmod 3$, since variables provide true values in multiples of 3 and each clause of $E^{\prime}$ absorbs one of these.

We now construct our final expression $E^{\prime \prime}$, and its graph $G^{\prime \prime}$, by gathering the additional occurrences around each face into a gadget as described in Section 3.3. This gadget will enforce that the correct number mod 3 of the additional occurrences are true. In what follows we sometimes write 0 and 1 for true and false respectively.

\subsection{Gadgets}

The purpose of a gadget is to be satisfiable provided the correct number mod 3, say $c$, of its $3 m$ input edges are true. Thus we complete the construction of $G^{\prime \prime}$ by placing a gadget in each face of $G^{\prime}$. 
A gadget is composed of a sequence of optional switches that allow the true and false values to be sorted in any order, and checked in groups of three. Specifically, we wish for the rightmost $c$ groups of three variables to have one true input each, and for all other groups of three variables to be either all true or all false. To do this, we terminate the rightmost $c$ groups with a single clause vertex, and all other groups with a 3-way equality verifier as defined below.

An optional switch has two entry edges and two exit edges. It can be satisfied in all cases where the two values on the exit edges are the same as the two values on the entry edges in either order. It uses two sub-gadgets, the partial switch which behaves the same way except that it cannot be satisfied if both entry edges are true, and the 3-way equality verifier, which requires all its inputs to have the same truth value.

The partial switch itself has a sub-gadget which we call a triangle. This is a component with three variable vertices $(a, b, c)$, each with one edge to the exterior, which requires that exactly one of $a, b$ and $c$ is true. In other words, it acts just like a clause, except that it uses two of each variable's occurrences. Note that we cannot achieve this simply by doubling the clause $(a, b, c)$ since this would violate planarity.

The triangle has three internal variables $a^{\prime}, b^{\prime}$ and $c^{\prime}$, and the clauses $\left(a, b, c^{\prime}\right)$, $\left(b, a^{\prime}, c^{\prime}\right),\left(c, a^{\prime}, b^{\prime}\right),\left(a, c, b^{\prime}\right)$ and $\left(a^{\prime}, b^{\prime}, c^{\prime}\right)$. This is satisfied by taking $a^{\prime}=a, b^{\prime}=b$ and $c^{\prime}=c$, and the central clause then ensures that exactly one of these is true. A triangle is shown in Fig. 16 together with the symbol used to denote it in larger components.

The partial switch is shown in Fig. 17. It has two input variables $p, q$ and two output variables $r, s$. It contains internal variables $a, b, c$ and $t$, a triangle $(a, b, c)$ and the clauses $(p, q, t),(b, r, t),(c, s, t),(a, r, s)$. If $p=q=0$, the partial switch is satisfied by taking $a=t=1$ and $b=c=r=s=0$. If $p \neq q$, we can take either $r=c=1$ and $a=b=s=t=0$ or $s=b=1$ and $a=c=r=t=0$, and in either case $r \neq s$. Thus the partial switch copies the two inputs to the two outputs in either order, except when both inputs are true.

Note that each partial switch uses two occurrences each of its input variables, and only one each of its output variables. Thus partial switches can be strung together in series. We use this to build the full optional switch shown in Fig. 18. This is composed of a new variable vertex (which may always be false) placed between the two entry edges
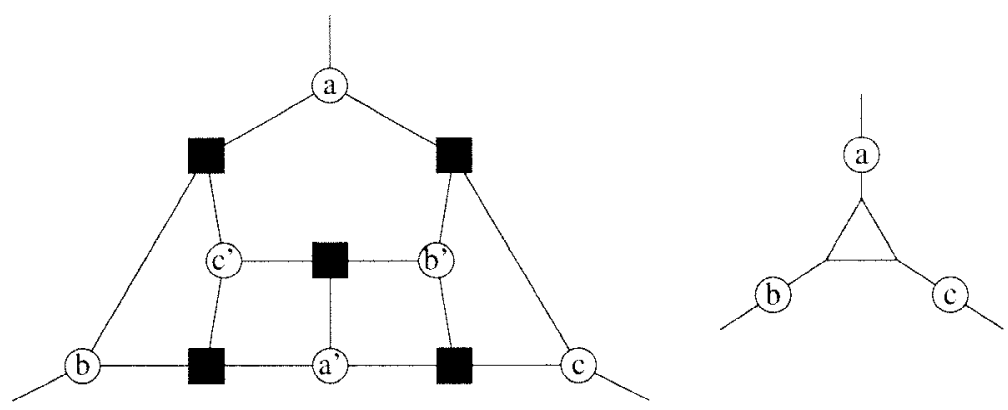

Fig. 16. A triangle and our abbreviation for it 

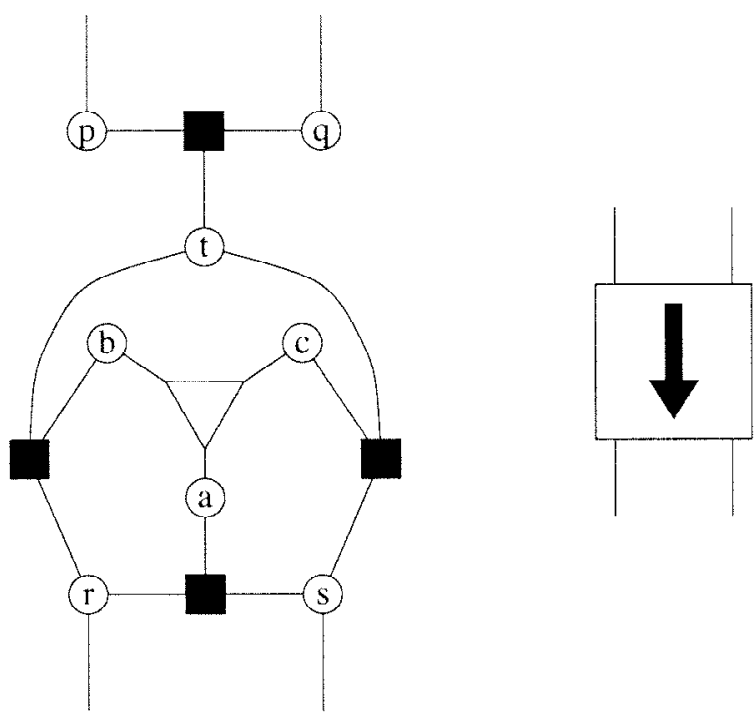

Fig. 17. A partial switch and its abbreviation.

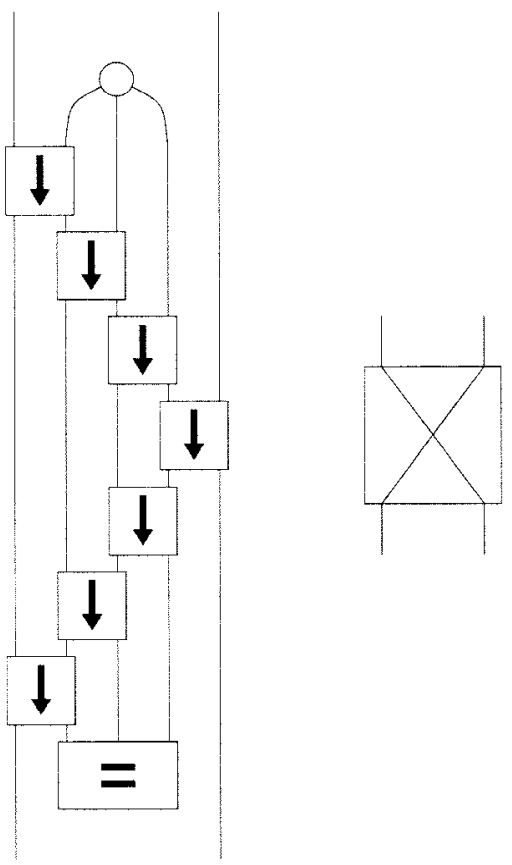

Fig. 18. An optional switch and its abbreviation 


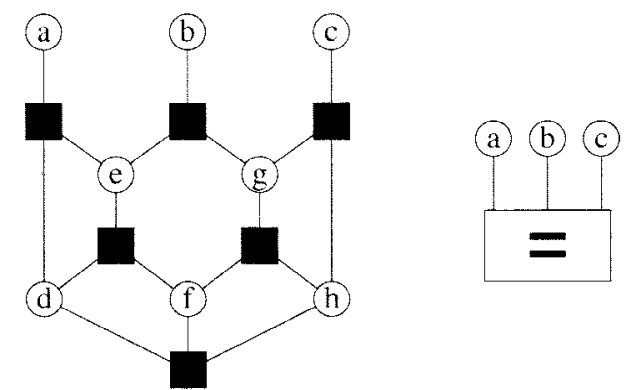

Fig. 19. A 3-way equality verifier and its abbreviation.

followed by two sequences of partial switches. These permit a single true value to be transmitted from entry 1 to exit 2 or vice versa. This leaves three edges between the two exit edges and these three edges are then passed into a 3-way equality verifier.

To complete our proof for Cubic Planar Monotone 1-in-3 SAT, we construct this verifier as in Fig. 19. It has three input edges from variables $a, b, c$ and five internal variables $d, e, f, g, h$. It contains the clauses $(a, d, e),(b, e, g),(c, g, h),(d, e, f),(f, g, h)$ and $(d, f, h)$. If $a=b=c=0$, we take either $d=g=1$ and $e=f=h=0$ or $e=h=1$ and $d=f=g=0$. If $a=b=c=1$ we take $f=1$ and $d=e=g=h=0$.

Finally, to construct a gadget for $3 m$ inputs of which $c \bmod 3$ are to be true, we form a sorting network from a polynomial number of optional switches that allows the inputs to be sorted in any order. We then divide the outputs of the sorting network into triples, and add a suitable checker to each triple. This checker will be a single clause for $c$ of the triples and a 3-way equality verifier for the rest. An example is shown in Fig. 20, where $m=2$ and $c=1$. In the case $m=1, c=2$, this construction needs a slight modification; we add a new variable and feed the six edges (the three inputs and the three outputs from the variable) into a $m=2, c=2$ gadget.

After the appropriate gadget is added to each face to absorb its additional occurrences, it is clear that $E^{\prime \prime}$ is satisfiable if and only if $E$ is. This completes our proof that Cubic Planar Monotone 1-in-3 Satisfiability, and therefore the existence problem for tilings by the right tromino, is NP-complete.

\section{Monotone Circuits in Three and Four Dimensions}

In this section we construct wires and gates in three dimensions with the domino and straight tromino, or (if you prefer) the dicube and straight tricube, shown in Fig. 21. The construction is similar to that in Section 2, except that it is limited to monotone Boolean circuits, whose outputs are non-decreasing functions of their inputs.

Our wires will consist of zigzags as in Fig. 22, which can be tiled by dominoes in either of two ways. As before, we give each wire an orientation. Then if we two-color the lattice as a three-dimensional checkerboard, dividing it into odd and even cells, we 


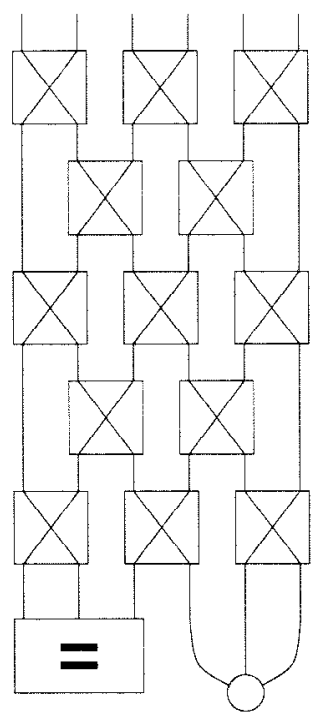

Fig. 20. A gadget which allows six inputs to be sorted and confirms that $1 \bmod 3$ of them are true.

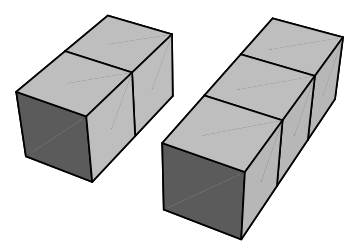

Fig. 21. The domino and straight tromino in three dimensions.

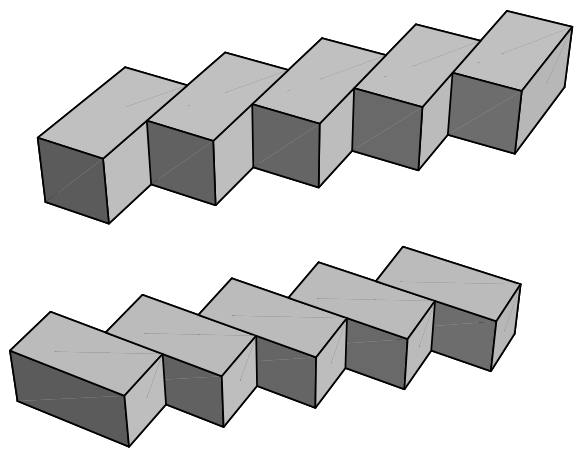

Fig. 22. The wire for tilings by the domino and straight tromino. It can be tiled by dominoes in either of two ways, such that the downstream end of each domino lies on either an odd cell (true) or an even cell (false). 


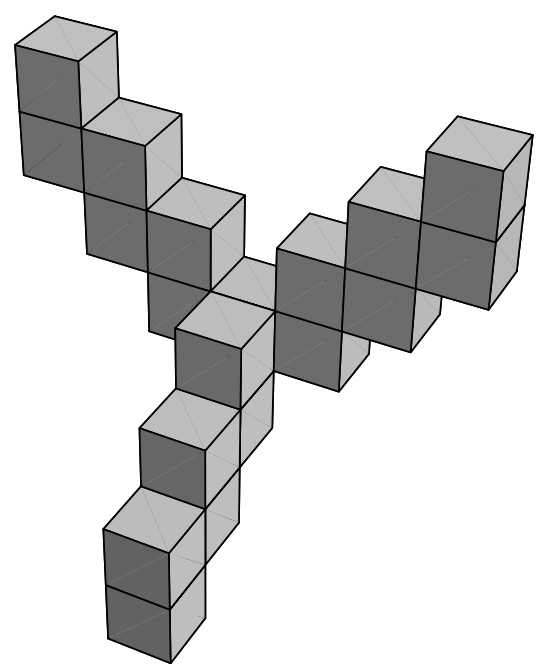

Fig. 23. This configuration serves as an AND or OR gate, depending on whether the central cell is even or odd.

define true and false wires as those where the downstream end of each domino lies on an odd or even cell respectively. Note that this allows wires to turn quite freely. Then the reader can easily check that the configuration in Fig. 23, with two inputs entering from above and an output exiting from below, acts as an AND gate if its central cell is even, and an OR gate if its central cell is odd. There is a unique tiling for each truth value of the inputs; in one of these four tilings the central crossbar is filled with a tromino, while the other tilings consist entirely of dominoes.

With AND and OR gates, we can build any monotone Boolean circuit. It seems to be difficult to build a NOT gate with these two tiles, and we conjecture that this is impossible. However, monotone circuits are enough for our purposes, since we can use De Morgan's laws $\overline{a \wedge b}=\bar{a} \vee \bar{b}$ and $\overline{a \vee b}=\bar{a} \wedge \bar{b}$ to move negations up through our gates [14], and thus convert any Boolean circuit with variables $x_{1}, \ldots, x_{n}$ to a monotone one with variables $x_{1}, \bar{x}_{1}, \ldots, x_{n}, \bar{x}_{n}$.

To make a one-to-one correspondence between satisfying assignments of this circuit and the original one, we just have to add the non-monotone condition that exactly one of $x_{i}$ and $\bar{x}_{i}$ be true for each $i$. We can do this by connecting these two variables with a zigzag wire; using our definition of orientation, this wire's tiling will come out as true at one end and false at the other. Finally, we require the output wire to be true by ending it on an odd cell.

To complete the argument, we need to be able to split wires. However, if we can do this, we can construct a NOT gate as shown in Fig. 24. Since our definition of truth is negated if we reverse the orientation of a wire, if we attach one output of a splitter backwards to a wire with value $x$, and attach its input to a variable that can be true or false, the other output will yield $\bar{x}$. Thus if we are correct that we cannot build a NOT gate, we cannot build a wire splitter either.

Since a perfect wire splitter does not seem to exist, we consider instead a "dirty splitter" where the outputs are equal to or less than the inputs; for instance, an OR gate 


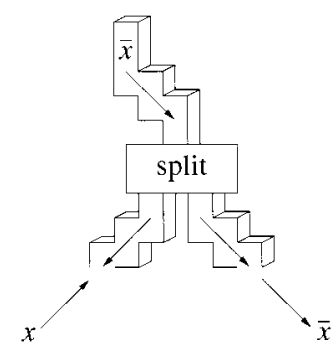

Fig. 24. If we had a wire splitter, we could make a NOT gate by feeding a wire with value $x$ into one of its outputs in reverse, and connecting its input into a region which can be tiled either true or false. Since our definition of truth value is negated if we reverse the orientation of a wire, the other output will then carry the negated value $\bar{x}$.

in reverse has this property. Since the circuit is monotone, this can create false negatives but not false positives, so this "dirty circuit" can have a true output if and only if the original circuit can.

Finally, standard arguments can be used to show that we can embed the required components in polynomially bounded 3-space with sufficient clearance between them so that the wires do not intersect. Thus we have shown how to convert a monotone Boolean circuit to a region of the cubic lattice that can be tiled with dominoes and straight trominoes if and only if the circuit is satisfiable, and the existence problem for such tilings is NP-complete.

We would like to say that the counting problem for such tilings is \#P-complete, but using dirty splitters means that in some cases there will be more than one tiling for a given satisfying assignment. Specifically, if a wire coming out of a dirty splitter is true but is not essential to making the output true, we can tile it false and the circuit will still be satisfied. Thus the mapping between assignments and tilings is not one-to-one, and our reduction from Satisfiability to the existence problem is not parsimonious. While it seems likely that counting these tilings is \#P-complete, it is not clear how to prove this.

It unlikely that the number of dimensions in this construction can be reduced from three to two. While planar circuits and monotone circuits can both simulate Boolean circuits in general [14], circuits which are both planar and monotone cannot.

Finally, we note that we can convert any finite region $R$ in the cubic lattice into a simply connected region $R^{\prime}$ in the four-dimensional hypercubic lattice with an equivalent tiling problem [17]. Simply choose a set of sites $S$ such that $R \cup S$ is simply connected, two-color them, and add each one along with an additional site adjacent to it in the fourth dimension, alternating up and down according to the two-coloring. Then these sites can only be filled by dominoes aligned along the fourth dimension, and only the sites in $R$ are left. Thus the tiling problem for dominoes and straight trominoes is also NP-complete for simply connected regions in the four-dimensional cubic lattice.

\section{Discussion}

For the right tromino and square tetromino on the square lattice, or the right tromino alone, we have shown that the existence problem for tiling regions of the square lattice is NP- 
complete. In the former case we have shown that the counting problem is \#P-complete. For the domino and straight tromino we have shown that the existence problem is NPcomplete for general regions in the cubic lattice, and for simply connected regions in the four-dimensional hypercubic lattice.

Intuitively, the NP-completeness of tiling with polyominoes with three or more cells comes from that fact that, while telling whether a graph can be covered with dimers is related to the Perfect Matching problem and can be solved in polynomial time, telling whether it can be covered by trimers is NP-complete [4]. While there is no strict connection between a system's computational complexity and its statistical mechanics, we might expect that tiling problems for tiles consisting of three or more cells are generally not exactly solvable in two or more dimensions (although the statistics of triangular trimers on the triangular lattice can be solved with a Bethe ansatz [18]).

For dominoes the existence and counting problems have an especially elegant solution. The number of ways to tile a region of a regular lattice with dominoes can be calculated by expressing it as a determinant of a modified adjacency matrix [19], [20]. This puts the existence and counting problems for the domino in the class DET $\subseteq \mathbf{P}$ and allows the statistics of the dimer model to be solved exactly.

It would be interesting to find other small sets of tiles for which tiling problems are hard, or find larger sets of tiles for which existence and/or counting are in $\mathbf{P}$. As noted above, in two dimensions the existence problem for dominoes and straight trominoes is in $\mathbf{P}$ when rotations are allowed [8], [9].

Another solvable case is that of a single polyomino without rotation, or one which is rotationally symmetric. Then there is at most one tiling of a given finite region, which we can find by scanning from left to right and top to bottom and adding a tile to each unoccupied cell we meet. Beauquier and Nivat [21] showed that this case of the tiling problem is decidable for the infinite plane as well. Finally, we note that the existence of an isohedral tiling of the infinite plane by a single polyomino-one whose symmetry group acts transitively on the set of tiles-was recently shown to be decidable by Keating and Vince [22].

Some open questions include:

1. Is the counting problem for the right tromino on the square lattice, or for the domino and straight tromino on the cubic lattice, \#P-complete?

2. Is the existence problem for the right tromino in $\mathbf{P}$ for simply connected regions in the square lattice?

3. Is the existence problem for dominoes and trominoes in $\mathbf{P}$ for simply connected regions in the cubic lattice?

\section{Acknowledgements}

We are grateful to Richard Kenyon, Michael Lachmann, Mark Newman, Mats Nordahl, Eric Rémila and Laurent Vuillon for helpful conversations. C.M. also thanks Robert Cori for an invitation to École Polytechnique, to Michel Morvan for an invitation to Université Paris 7 (Jussieu) where the authors met, and to Molly Rose and Spootie the Cat for warmth and friendship. 


\section{References}

1. R. Berger, The undecidability of the domino problem. Mem. Amer. Math. Soc. 66 (1966), 1-72.

2. R.M. Robinson, Undecidability and nonperiodicity of tilings of the plane. Invent. Math. 12 (1971), 177209.

3. H. Lewis, Complexity of solvable cases of the decision problem for predicate calculus. Proc. 19th Symp. Foundations of Computer Science (1978), pp. 35-47.

4. M.R. Garey and D.S. Johnson, Computers and Intractability: A Guide to the Theory of NP-Completeness. Freeman, San Francisco, CA, 1979.

5. S.W. Golomb, Polyominoes: Puzzles, Patterns, Problems, and Packings, revised edition. Princeton University Press, Princeton, NJ, 1994.

6. D. Beauquier, M. Nivat, E. Rémila and J.M. Robson, Comput. Geom. 5 (1995), 1-25.

7. C. Kenyon and R. Kenyon, Tiling a polygon with rectangles. Proc. 33rd Symp. Foundations of Computer Science (1992), pp. 610-619.

8. E. Rémila, Tiling a simply connected figure with bars of length 2 and length 3. Discrete Math. 160 (1996), 189-198.

9. E. Rémila, Tilings with bars and satisfaction of Boolean formulas. European J. Combin. 17 (1996), 485491.

10. C.H. Papadimitriou, Computational Complexity. Addison-Wesley, Reading, MA, 1994.

11. E. Goles and I. Rapaport, Complexity of tile rotation problems. Theoret. Comput. Sci. 188(1997), 129-159.

12. E. Goles and I. Rapaport, Tiling allowing rotations only. Theoret. Comput. Sci. 218 (1999), 285-295.

13. K. Lindgren, C. Moore and M.G. Nordahl, Complexity of two-dimensional patterns. J. Statist. Phys. 91 (1998), 909-951.

14. L.M. Goldschlager, The monotone and planar circuit value problems are log space complete for P. SIGACT News 9 (1977), 25-29.

15. G. Di Battista, P. Eades, R. Tamassia and I.G. Tollis, Graph Drawing, Prentice Hall, Englewood Cliffs, NJ, 1999.

16. P. Laroche, Planar 1-in-3 satisfiability is NP-complete. ASMICS Workshop on Tilings, Deuxième Journées Polyominos et pavages, Ecole Normale Supérieure de Lyon, 1992.

17. R. Kenyon, Personal communication.

18. A. Verberkmoes and B. Nienhuis, Triangular trimers on the triangular lattice: an exact solution. cond-mat/9904343

19. P.W. Kasteleyn, The statistics of dimers on a lattice, I: The number of dimer arrangements on a quadratic lattice. Physica 27 (1961), 1209-1225.

20. J. Propp, Dimers and dominoes. Manuscript available at http://www-math.mit.edu/ propp/ articles. html.

21. D. Beauquier and M. Nivat, On translating one polyomino to tile the plane. Discrete Comput. Geom. 6 (1991), 575-592

22. K. Keating and A. Vince, Isohedral polyomino tiling of the plane. Discrete Comput. Geom. 21 (1999), $615-630$.

Received March 8, 2000, and in revised form May 14, 2001, and June 18, 2001.

Online publication October 12, 2001. 